\title{
Parallel PWTD-Accelerated Explicit Solution of the Time Domain Electric Field Volume Integral Equation
}

\author{
Yang Liu, Member, IEEE, Ahmed Al-Jarro, Member, IEEE, Hakan Bağc1, Senior Member, IEEE, Eric \\ Michielssen, Fellow, IEEE
}

\begin{abstract}
A parallel plane-wave time-domain (PWTD)-accelerated explicit marching-on-in-time (MOT) scheme for solving the time domain electric field volume integral equation (TD-EFVIE) is presented. The proposed scheme leverages pulse functions and Lagrange polynomials to spatially and temporally discretize the electric flux density induced throughout the scatterers, and a finite difference scheme to compute the electric fields from the Hertz electric vector potentials radiated by the flux density. The flux density is explicitly updated during time marching by a predictor-corrector (PC) scheme and the vector potentials are efficiently computed by a scalar PWTD scheme. The memory requirement and computational complexity of the resulting explicit PWTD-PC-EFVIE solver scale as $O\left(N_{s} \log N_{s}\right)$ and $O\left(N_{s} N_{t}\right)$, respectively. Here, $N_{s}$ is the number of spatial basis functions and $N_{t}$ is the number of time steps. A scalable parallelization of the proposed MOT scheme on distributed-memory CPU clusters is described. The efficiency, accuracy, and applicability of the resulting (parallelized) PWTD-PC-EFVIE solver are demonstrated via its application to the analysis of transient electromagnetic wave interactions on canonical and real-life scatterers represented with up to 25 million spatial discretization elements.
\end{abstract}

Index Terms-Time domain electric field volume integral equation (TD-EFVIE), plane-wave time-domain algorithm (PWTD), predictor-corrector scheme, explicit marching-on-in-time (MOT) scheme, large-scale problems, transient analysis.

Manuscript received August 31, 2015. This work was supported in part by the AFOSR/NSSEFF Program under Award FA9550-10-1-0180 and the National Science Foundation (NSF) under Grant CCF 1116082.

Y. Liu and E. Michielssen are with the Department of Electrical Engineering and Computer Science, University of Michigan, Ann Arbor, MI 48109, USA (e-mails: liuyangz@umich.edu,emichiel@umich.edu).

A. Al-Jarro was with the Division of Computer, Electrical, and Mathematical Sciences and Engineering, King Abdullah University of Science and Technology (KAUST), Thuwal 23955-6900, Saudi Arabia. He is now with the Department of Electronic and Electrical Engineering, University College London, London WC1E7JE, UK (e-mail: ahmed.aljarro@ucl.ac.uk).

H. Bağcı is with the Division of Computer, Electrical, and Mathematical Sciences and Engineering King Abdullah University of Science and Technology (KAUST), Thuwal 23955-6900, Saudi Arabia. He is also with the Center for Uncertainty Quantification in Computational Science and Engineering at KAUST (e-mail: hakan.bagci@kaust.edu.sa).

\section{INTRODUCTION}

$\mathrm{T}$ ransient analysis of electromagnetic wave interactions on electrically large inhomogeneous dielectric scatterers is called for in various applications of engineering and science ranging from the design of optoelectronic devices and broadband antenna radomes to the study of (un)intentional radiation effects on human tissue/cells [1,2]. Among simulators capable of electromagnetic characterization of such scatterers, time domain electric field volume integral equation (TD-EFVIE) solvers are rapidly gaining ground [3-7]. The TD-EFVIE is constructed by enforcing that the total electric field is equal to the incident electric field plus the scattered electric field radiated by the electric flux density induced throughout the scatterer. To numerically solve the TD-EFVIE, the unknown electric flux density is expanded in terms of spatio-temporal basis functions. Inserting this expansion into the TD-EFVIE and testing the resulting equation in space and time yield a set of linear systems that can be solved for the unknown expansion coefficients typically by marching-on-in-time (MOT).

The MOT scheme can be implicit or explicit, depending on the types of the spatio-temporal basis expansion and testing scheme and the size of the time step. The implicit MOT scheme require at every time step solution of the linear system [3], which is traditionally constructed upon expanding the flux density with Schaubert-Wilton-Glisson (SWG) spatial basis functions [8] and piecewise polynomial temporal basis functions [9], followed by Galerkin and point testing in space and time, respectively. In addition, modern implicit MOT-based solution of time domain surface and volume integral equations can be made low- and high-frequency stable by using computationally more expensive space-time discretization techniques, such as bandlimited time discretization [7, 10], space-time Galerkin testing $[11,12]$, quasi-Helmholtz decomposition [13, 14], and highly accurate evaluation of MOT matrix elements [12, 15-20]. In contrast, the explicit MOT scheme, usually leverages pulse spatial basis functions and low order temporal basis functions and point testing both in space and time. These "simplifications" render the explicit MOT scheme computationally more efficient, but yet less stable than its implicit counterpart [21]. This problem has been alleviated by a recently 
developed explicit MOT-TD-EFVIE solver, which leverages a predictor-corrector (PC) scheme to "stabilize" updates of the flux density during time marching [22]. Moreover, CPU parallelized [23] and GPU accelerated [24] implementations are developed to further advance the capability of the solver. That said, the applicability of this solver to the real-life scattering problems is still limited by its high computational complexity, i.e., $O\left(N_{s}^{2} N_{t}\right)$ where $N_{s}$ is the number of spatial basis functions and $N_{t}$ is the number of simulation time steps. To overcome this bottleneck, fast algorithms such as multilevel plane-wave time-domain algorithm (PWTD) [25] and time-domain adaptive integral method (TD-AIM) [26] must be considered.

In this work, a (parallelized) PWTD-accelerated PC-based MOT-TD-EFVIE solver is developed. In the past, PWTD has been successfully used for accelerating the MOT-based solution of time domain surface and volume integral equations $[4,5$, 25]. When used in tandem with TD-EFVIE solvers, it reduces the abovementioned computational complexity to $O\left(N_{s} N_{t}\right)$ [4, 5]. Previously, various PWTD-accelerated implicit MOT-TD-EFVIE solvers have been developed wherein the PWTD scheme permits fast computation of scattered electric fields due to past flux density [4, 5]. However, the explicit MOT-TD-EFVIE solver computes the scattered electric fields from vector potentials using a finite difference scheme. Hence, the PWTD scheme proposed here, unlike its previously developed versions, is used for accelerating the computation of the vector potentials due to the flux density. More specifically, at the predictor step of the time marching algorithm, the interactions between far field box pairs are evaluated by decomposing vector potentials into their three Cartesian components, which are propagated independently using a scalar field PWTD scheme. Vector potential contribution from near field boxes is computed as it is. Then, a finite difference scheme is applied to the samples of the vector potential to "predict" the electric fields. At the corrector step, the electric fields are updated (i.e., corrected) using a time-dependent averaging factor that improves the accuracy while maintaining the stability (as opposed to stabilization scheme that leverages a constant averaging factor as proposed in [22]). Furthermore, a scalable CPU parallelized implementation of the proposed PWTD-PC-EFVIE solver is also described. The efficiency and accuracy of the solver are demonstrated via its application to analysis of transient scattering from canonical objects represented with up to 25 million spatial discretization elements. Furthermore, the solver is applied to characterization of light interaction with a red blood cell aggregation discretized with 12 million spatial elements.

The rest of the paper is organized as follows. Section II-A formulates the PC-based MOT scheme for solving the TD-EFVIE. Section II-B delineates the scalar field PWTD algorithm used for accelerating the PC-based MOT scheme. Section III describes the parallelization of the PWTD-PC-EFVIE solver on distributed-memory CPU clusters. Numerical examples, which demonstrate the efficiency, accu- racy, and applicability of the proposed parallelized PWTD-PC-EFVIE solver, are presented in Section IV, followed by the concluding remarks in Section V.

\section{FORMULATION}

\section{A. PC-Based MOT Scheme for TD-EFVIE Solution}

Let $V$ denote the support of inhomogeneous dielectric scatterers that reside in an unbounded background medium with permittivity $\varepsilon_{b}$. It is assumed that the scatterers are isotropic, non-magnetic, non-dispersive, lossless, and have permittivity $\varepsilon(\mathbf{r})$. Let $\mathbf{E}^{i}(\mathbf{r}, t)$ denote an incident electric field that is (essentially) bandlimited to maximum frequency $f_{\max }$. It is assumed that $\mathbf{E}^{i}(\mathbf{r}, t)=0, \forall \mathbf{r} \in V$ for $t<0$. Upon excitation of the scatterers by $\mathbf{E}^{i}(\mathbf{r}, t)$, an electric flux density $\varepsilon(\mathbf{r}) \mathbf{E}(\mathbf{r}, t)$ is induced on $V$. This electric flux density generates a scattered electric field $\mathbf{E}^{s}(\mathbf{r}, t)$. Here, $\mathbf{E}(\mathbf{r}, t)$ is the total electric field. A TD-EFVIE can be formulated by decomposing $\mathbf{E}(\mathbf{r}, t)$ into $\mathbf{E}^{i}(\mathbf{r}, t)$ and $\mathbf{E}^{s}(\mathbf{r}, t)$ as

$$
\begin{aligned}
\mathbf{E}(\mathbf{r}, t) & =\mathbf{E}^{i}(\mathbf{r}, t)+\mathbf{E}^{s}(\mathbf{r}, t) \\
& =\mathbf{E}^{i}(\mathbf{r}, t)+\nabla \nabla \cdot \mathbf{P}(\mathbf{r}, t)-\frac{\partial_{t}^{2}}{c_{b}^{2}} \mathbf{P}(\mathbf{r}, t) .
\end{aligned}
$$

Here, $c_{b}$ is the speed of light in the background medium, $\partial_{t}$ denotes time derivative, and $\mathbf{P}(\mathbf{r}, t)$ represents the Hertz electric vector potential due to the induced electric flux density and is expressed as

$$
\mathbf{P}(\mathbf{r}, t)=\int_{V} d \mathbf{r}^{\prime} \frac{\left(\varepsilon\left(\mathbf{r}^{\prime}\right)-\varepsilon_{b}\right) \mathbf{E}\left(\mathbf{r}^{\prime}, t-R / c_{b}\right)}{4 \pi \varepsilon_{b} R} .
$$

Here, $R=\left|\mathbf{r}-\mathbf{r}^{\prime}\right|$ is the distance between source point $\mathbf{r}^{\prime}$ and observer point $\mathbf{r}$.

To numerically solve (1), the computation domain is discretized using cubic elements with edge length $\Delta s$. It is assumed that $\Delta s$ resolves the minimum wavelength inside the scatterers and the permittivity inside the $n$th element is constant, i.e., $\varepsilon(\mathbf{r})=\varepsilon\left(\mathbf{r}_{n}\right), \mathbf{r} \in V_{n}$, where $\mathbf{r}_{n}$ is the center and $V_{n}$ is the support of the $n$th element. Next, the total electric field in the scatterers, $\mathbf{E}(\mathbf{r}, t)$, is discretized using spatial basis functions $S_{n}(\mathbf{r}), n=1, \ldots, N_{s}$ and temporal basis functions $T_{j}(t)$, $j=1, \ldots, N_{t}$, as

$$
\mathbf{E}(\mathbf{r}, t)=\sum_{n=1}^{N_{s}} \sum_{j=1}^{N_{t}} \mathbf{E}_{n, j} S_{n}(\mathbf{r}) T_{j}(t) .
$$

Here, $\mathbf{E}_{n, j}$ is the vector expansion coefficient (with three Cartesian components) associated with the spatio-temporal basis function $S_{n}(\mathbf{r}) T_{j}(t), S_{n}(\mathbf{r})$ is the pulse basis function defined in the $n$th cubic element, i.e., $S_{n}(\mathbf{r})=1, \mathbf{r} \in V_{n}$, $T_{j}(t)=T(t-j \Delta t)$ is the shifted Lagrange polynomial [9], and $\Delta t$ is the time step size defined by a Courant-Friedrichs-Lewy (CFL)-type condition, as $c_{b} \Delta t \leq \Delta s \leq 2 c_{b} \Delta t$. With this choice of spatio-temporal basis function, the expansion coefficient $\mathbf{E}_{n, j}$ simply becomes the electric field sampled at the $n$th element center and $j$ th time step, i.e., $\mathbf{E}_{n, j}=\mathbf{E}\left(\mathbf{r}_{n}, t_{j}\right)=\mathbf{E}\left(\mathbf{r}_{n}, j \Delta t\right)$. 


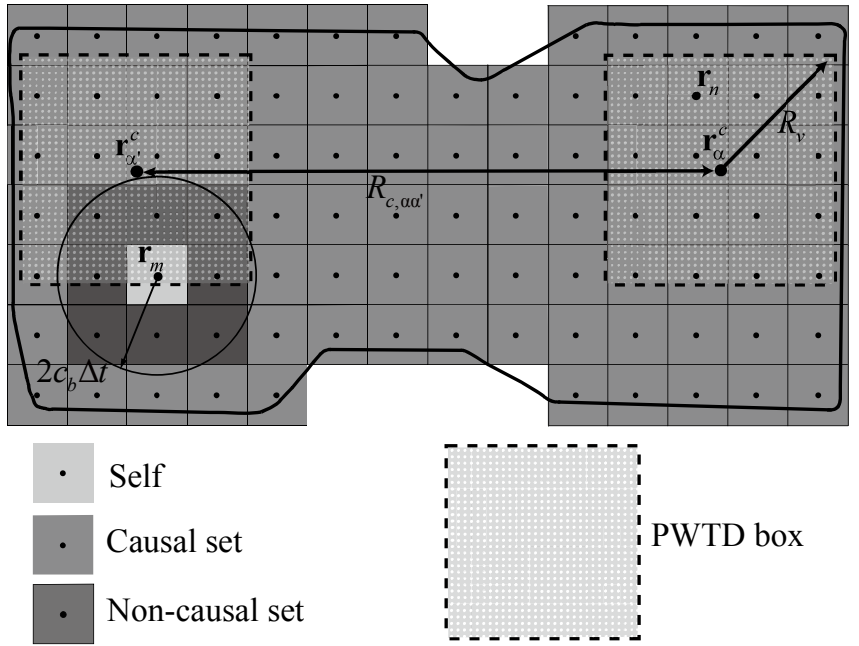

Fig. 1: A H-shaped structure discretized using cubic (source) elements. One far field box pair is shown in the figure.

To compute $\mathbf{E}_{n, j}$ via explicit MOT, (1) is enforced at space-time samples $\left(\mathbf{r}_{m}, t_{i}\right), m=1, \ldots, N_{s}, i=1, \ldots, N_{t}$, and the spatial operator $\nabla \nabla \cdot$ and temporal operator $\partial_{t}^{2} / c_{b}^{2}$ in (1) are approximated by a finite difference scheme. Note that this scheme requires the computation of the vector potential $\mathbf{P}(\mathbf{r}, t)$ at times $t_{i-1}, t_{i}, t_{i+1}$, and at element centers $\mathbf{r}_{m}, m=1, \ldots, N_{s}^{\prime}$. Here, $N_{s}^{\prime}=N_{s}+N_{s}^{b}$ and $N_{s}^{b}$ denotes the number of elements in the background medium with at least one adjacent element residing in the scatterers. It should also be noted here that the element indices are sorted such that the first $N_{s}$ elements correspond to those in the scatterers and the following $N_{s}^{b}$ elements correspond to those in the background medium. Upon substituting (3) into (2), the vector potential sample $\mathbf{P}\left(\mathbf{r}_{m}, t_{i}\right)$, $m=1, \ldots, N_{s}^{\prime}$ is computed as

$$
\mathbf{P}\left(\mathbf{r}_{m}, t_{i}\right)=\mathbf{P}_{s}\left(\mathbf{r}_{m}, t_{i}\right)+\mathbf{P}_{c}\left(\mathbf{r}_{m}, t_{i}\right)+\mathbf{P}_{n c}\left(\mathbf{r}_{m}, t_{i}\right) .
$$

The first term $\mathbf{P}_{s}\left(\mathbf{r}_{m}, t_{i}\right)$ on the right hand side (RHS) of (4) is dubbed the "self-term" contribution and is computed by

$$
\begin{aligned}
& \mathbf{P}_{s}\left(\mathbf{r}_{m}, t_{i}\right)=\left(\varepsilon\left(\mathbf{r}_{m}\right)-\varepsilon_{b}\right) \beta \mathbf{E}_{m, i} \\
& \beta=\int_{V_{m}} d \mathbf{r} S_{m}(\mathbf{r}) /\left(4 \pi \varepsilon_{b}\left|\mathbf{r}-\mathbf{r}_{m}\right|\right) .
\end{aligned}
$$

Note that $\beta$ is a constant independent of $m$ and can be computed analytically [27]. The second/third term $\mathbf{P}_{r}\left(\mathbf{r}_{m}, t_{i}\right)$, $r \in\{c, n c\}$ on the RHS of (4) is computed by

$$
\mathbf{P}_{r}\left(\mathbf{r}_{m}, t_{i}\right)=\sum_{n \in r_{m}} \sum_{j \in I_{i m n}} \frac{w_{n} \mathbf{E}_{n, j} T_{j-i}\left(-R_{m n} / c_{b}\right)}{R_{m n}} .
$$

Here, $\quad w_{n}=\Delta s^{3}\left(\varepsilon\left(\mathbf{r}_{n}\right)-\varepsilon_{b}\right) /\left(4 \pi \varepsilon_{b}\right) \quad, \quad R_{m n}=\left|\mathbf{r}_{m}-\mathbf{r}_{n}\right|$, $I_{i m n}=\left\{i-\left\lfloor R_{m n} / c_{b} \Delta t\right\rfloor, \ldots, i-\left\lfloor R_{m n} / c_{b} \Delta t\right\rfloor-p\right\}$, and $p$ denotes the order of the temporal basis function. Note that to derive (7), the single point quadrature rule is used for the integration in (2). In (7), $c_{m}$ and $n c_{m}$ denote the "causal" and "non-causal" set of source points for observation point $\mathbf{r}_{m}$. Specifically, $c_{m}=\left\{n:\left|\mathbf{r}_{m}-\mathbf{r}_{n}\right| \geq 2 c_{b} \Delta t\right\}$ and the computation of
$\mathbf{P}_{c}\left(\mathbf{r}_{m}, t_{i+1}\right)$ does not require the knowledge of $\mathbf{E}_{n, j}$ at time steps $j \geq i$. In contrast, $n c_{m}=\left\{n:\left|\mathbf{r}_{m}-\mathbf{r}_{n}\right|<2 c_{b} \Delta t, m \neq n\right\}$ and the computation of $\mathbf{P}_{n c}\left(\mathbf{r}_{m}, t_{i+1}\right)$ requires the knowledge of $\mathbf{E}_{n, i}$, which is not yet available. For this reason, $\mathbf{P}_{c}$ and $\mathbf{P}_{n c}$ represent the "causal" and "non-causal" contributions, respectively. As an example, a H-shaped structure is discretized by cubic elements with their element centers represented by small dots (Fig. 1). Consider an observer element $m, m$ resides inside or outside the scatterer if $m \leq N_{s}$ or $N_{s}<m<N_{s}^{\prime}$. The self element and causal/non-causal set of source elements are shown in Fig 1. Under the CFL condition, the size of the non-causal sets is relatively small.

In what follows, the proposed explicit MOT scheme for solving (1) is elucidated. At each time step $i$, the electric field sampled at the $m$ th element center, $\mathbf{E}\left(\mathbf{r}_{m}, t_{i}\right)$, is computed by a predictor-corrector scheme. First, the predictor step estimates a total electric field throughout the scatterers, $\mathbf{E}^{\text {pre }}\left(\mathbf{r}_{m}, t_{i}\right)$, without knowledge of the unknown quantities $\mathbf{P}_{s}\left(\mathbf{r}_{m}, t_{i}\right)$ and $\mathbf{P}_{n c}\left(\mathbf{r}_{m}, t_{i+1}\right)$, by

$$
\begin{aligned}
\mathbf{E}^{\text {pre }}\left(\mathbf{r}_{m}, t_{i}\right) & =\beta_{m}\left\{\mathbf{E}^{i}\left(\mathbf{r}_{m}, t_{i}\right)-\tilde{\boldsymbol{\delta}}^{b}\left[\mathbf{P}_{s}\right]\left(\mathbf{r}_{m}, t_{i}\right)\right. \\
& -\delta^{c}\left[\mathbf{P}_{c}\right]\left(\mathbf{r}_{m}, t_{i}\right)-\delta^{b}\left[\mathbf{P}_{n c}\right]\left(\mathbf{r}_{m}, t_{i}\right) \\
& \left.+\mathcal{G}\left[\mathbf{P}_{c}+\mathbf{P}_{n c}\right]\left(\mathbf{r}_{m}, t_{i}\right)+\mathcal{G}\left[\mathbf{P}_{s}\right]\left(\mathbf{r}_{m}, t_{i-1}\right)\right\}
\end{aligned}
$$

where $\beta_{m}=1 /\left\{1+2 \beta\left(\varepsilon\left(\mathbf{r}_{m}\right)-\varepsilon_{b}\right) /\left(c_{b}^{2} \Delta t^{2}\right)\right\}$. Here, $\delta^{c}[\cdot]$ and $\delta^{b}[\cdot]$ are the second order central and backward difference approximations of the temporal operator $\partial_{t}^{2} / c_{b}^{2}$ and are expressed as

$\delta^{c}[f]\left(t_{i}\right)=\left\{f\left(t_{i+1}\right)-2 f\left(t_{i}\right)+f\left(t_{i-1}\right)\right\} /\left(c_{b}^{2} \Delta t^{2}\right)$,

$\delta^{b}[f]\left(t_{i}\right)=\left\{2 f\left(t_{i}\right)-5 f\left(t_{i-1}\right)+4 f\left(t_{i-2}\right)-f\left(t_{i-3}\right)\right\} /\left(c_{b}^{2} \Delta t^{2}\right)$

and $\tilde{\delta}^{b}[f]\left(t_{i}\right)=\delta^{b}[f]\left(t_{i}\right)-2 f\left(t_{i}\right) /\left(c_{b}^{2} \Delta t^{2}\right)$. In (8), $\mathcal{G}[\cdot]$ is the first order central difference approximation of the spatial operator $\nabla \nabla \cdot$, which can be found in Appendix of [22].

Next, the corrector step uses $\mathbf{E}^{\text {pre }}\left(\mathbf{r}_{m}, t_{i}\right)$ to update the self-term and non-causal potential contributions that, in turn, are used to correct the total electric field. The predicted self-term contribution $\mathbf{P}_{s}^{\text {pre }}\left(\mathbf{r}_{m}, t_{i}\right)$ is now computed using $\mathbf{E}^{\text {pre }}\left(\mathbf{r}_{m}, t_{i}\right)$ instead of $\mathbf{E}_{m, i}$ in (5); the predicted non-causal contribution at time step $i+1, \mathbf{P}_{n c}^{\text {pre }}\left(\mathbf{r}_{m}, t_{i+1}\right)$, is computed using $\mathbf{E}^{\text {pre }}\left(\mathbf{r}_{n}, t_{i}\right)$ instead of $\mathbf{E}_{n, i}$ in (7) [together with $\mathbf{E}_{n, j}$ that are computed at the previous time steps and satisfy the conditions required by (7)]. It should be re-emphasized here that the computations of $\mathbf{P}_{s}^{\text {pre }}\left(\mathbf{r}_{m}, t_{i}\right)$ and $\mathbf{P}_{n c}^{\text {pre }}\left(\mathbf{r}_{m}, t_{i+1}\right)$ are localized in space and time since they only require the computation of vector potential contributions from the field samples at $\mathbf{r}_{n}$ satisfying $\left\{n:\left|\mathbf{r}_{m}-\mathbf{r}_{n}\right|<2 c_{b} \Delta t\right\}$ for a duration of $p$ time steps. The electric field, $\mathbf{E}\left(\mathbf{r}_{m}, t_{i}\right)$, is then corrected/updated using 


$$
\begin{aligned}
\mathbf{E}\left(\mathbf{r}_{m}, t_{i}\right)= & \beta_{m}\left\{\mathbf{E}^{i}\left(\mathbf{r}_{m}, t_{i}\right)-\tilde{\delta}^{b}\left[\mathbf{P}_{s}^{p r e}\right]\left(\mathbf{r}_{m}, t_{i}\right)\right. \\
& -\omega_{m i} \delta^{c}\left[\mathbf{P}_{c}+\mathbf{P}_{n c}^{p r e}\right]\left(\mathbf{r}_{m}, t_{i}\right) \\
& -\left(1-\omega_{m i}\right) \delta^{c}\left[\mathbf{P}_{c}+\mathbf{P}_{n c}\right]\left(\mathbf{r}_{m}, t_{i-1}\right) \\
& +\omega_{m i} \mathcal{G}\left[\mathbf{P}_{s}^{p r e}+\mathbf{P}_{c}+\mathbf{P}_{n c}^{p r e}\right]\left(\mathbf{r}_{m}, t_{i}\right) \\
& \left.+\left(1-\omega_{m i}\right) \mathcal{G}\left[\mathbf{P}_{s}+\mathbf{P}_{c}+\mathbf{P}_{n c}\right]\left(\mathbf{r}_{m}, t_{i-1}\right)\right\} .
\end{aligned}
$$

Note that the central difference $\delta^{c}[\cdot]$ is now applied to both the causal and non-causal contributions. In (11), $\omega_{m i}$ denotes an averaging factor that stabilizes the corrector step. Throughout this paper, it is assumed that the incident electric field is a plane wave propagating in direction $\hat{\mathbf{k}}$. In this case, $\omega_{m i}$ is chosen as

$$
\omega_{m i}=\left\{\begin{array}{c}
1, t_{i}<\tau_{m}+\tau_{1} \\
1+\frac{1}{2} \cos \left(\frac{\pi\left(t_{i}-\tau_{m}-\tau_{1}\right)}{2\left(\tau_{2}-\tau_{1}\right)}\right), \tau_{m}+\tau_{1} \leq t_{i} \leq \tau_{m}+\tau_{2} . \\
0.5, t_{i}>\tau_{m}+\tau_{2}
\end{array}\right.
$$

Here, $\tau_{m}=\hat{\mathbf{k}} \cdot \mathbf{r}_{m} / c_{b}$, and $\tau_{2}>\tau_{1}>0$ are parameters related to the bandwidth of the plane wave. Note that larger $\omega_{m i}$ leads to better accuracy, yet worse stability, and $\omega_{m i}=1$ means that that no averaging is applied. This method of selecting $\omega_{m i}$ ensures that it varies smoothly as a function of $m$ and $i$, and stabilizes the MOT solution in the "late time". Once $\mathbf{E}\left(\mathbf{r}_{m}, t_{i}\right)$ is computed (and stored to be used in the next time steps), the vector potential contributions $\mathbf{P}_{s}\left(\mathbf{r}_{m}, t_{i}\right)$ and $\mathbf{P}_{n c}\left(\mathbf{r}_{m}, t_{i+1}\right)$ are updated, and the MOT scheme moves to the next time step $i+1$.

The computational and memory costs of the PC-based MOT scheme described above depend on those associated with the computation of the electric fields using the finite differences in (8)/(11) and computation of the vector potentials using (7). The former requires only space-time localized operations and is computationally inexpensive. The latter, in contrast, involves interactions between $N_{s}^{\prime}=O\left(N_{s}\right)$ observer points and $N_{s}$ source points for $N_{t}$ time steps, and requires storage of $\mathbf{E}_{n, j}$ at $N_{s}$ source points for $O\left(D_{\max } /\left[c_{b} \Delta t\right]\right)=O\left(N_{s}^{1 / 3}\right)$ consecutive time steps. Here, $D_{\max }$ is the maximum of any possible distance between all source and observer points. As a result, the computational and memory costs of the latter (and the solver) scale as $O\left(N_{t} N_{s}^{2}\right)$ and $O\left(N_{s}^{4 / 3}\right)$, respectively. These costs are prohibitively high when the solver is applied to the analysis of transient phenomena involving electrically large scatterers. Next, a PWTD-accelerated PC-based MOT scheme that requires only $O\left(N_{t} N_{s}\right)$ CPU and $O\left(N_{s} \log N_{s}\right)$ memory resources is described.

\section{B. PWTD Acceleration}

The PWTD algorithm described in this section permits fast evaluation of the vector potential $\mathbf{P}_{r}\left(\mathbf{r}_{m}, t_{i+1}\right)$ in (7), rather than evaluation of the (scattered) electric fields generated by the flux density as in the PWTD-accelerated implicit MOT-based TD-EFVIE solvers $[4,5]$. In what follows, the PWTD algorithm is briefly summarized while only those details pertinent to the aforementioned differences are provided.

First, a fictitious box enclosing the $N_{s}^{\prime}$ elements is recur- sively subdivided into eight smaller boxes until the edge length of the smallest boxes reaches a prescribed portion of the minimum wavelength at the maximum frequency, $\lambda=c_{b} / f_{\max }$. An element $n$ is said to reside in a box if its center $\mathbf{r}_{n}$ is located inside that box; empty boxes are immediately discarded. This procedure constructs a hierarchical tree structure of $N_{L}=O\left(\log \left[N_{s}^{1 / 3}\right]\right)$ levels. At each level $v, v=1, \ldots, N_{L}$, there exist $N_{g}^{v} \approx 8^{N_{L}-v}$ nonempty boxes. The radius of a sphere enclosing a level $v$ box is $R^{v}=2^{(v-1)} R^{1}$ with $R^{1}=O(1)$.

Next, starting from the coarsest level $N_{L}$, two boxes $\alpha$ and $\alpha^{\prime}$ centered at $\mathbf{r}_{\alpha}^{c}$ and $\mathbf{r}_{\alpha^{\prime}}^{c}$ are termed a far field pair if (i) the distance between their centers $R_{c, \alpha \alpha^{\prime}}=\left|\mathbf{R}_{c, \alpha \alpha^{\prime}}\right|=\left|\mathbf{r}_{\alpha^{\prime}}^{c}-\mathbf{r}_{\alpha}^{c}\right|$ satisfies the condition $R_{c, \alpha \alpha^{\prime}}>\gamma R^{v},(4 \leq \gamma \leq 6)$, and (ii) their parent boxes do not constitute a far field pair. Those box pairs at the finest level $v=1$, which do not constitute far field pairs, are termed near field pairs. Also, each box at the finest level forms a near field pair with itself. The interactions between elements in near field pairs (self interaction excluded) henceforth called near field calculation- are directly evaluated by (7), while those between far field pairs are handled by the PWTD scheme. When the finest level box size is properly chosen, all non-causal contribution $\mathbf{P}_{n c}\left(\mathbf{r}_{m}, t_{i+1}\right)$ and partial causal contribution $\mathbf{P}_{c}\left(\mathbf{r}_{m}, t_{i+1}\right)$ are handled by the near field calculation; the rest causal contribution $\mathbf{P}_{c}\left(\mathbf{r}_{m}, t_{i+1}\right)$ is accounted for by PWTD (see the example in Fig. 1).

Consider a far field pair $\alpha$ and $\alpha^{\prime}$ (Fig. 1), the total electric field at the $n$th source element in box $\alpha, \mathbf{E}\left(\mathbf{r}_{n}, t\right)$, is first represented using an approximate prolate spheroidal (APS) function $T^{A P S}(t)$ that is bandlimited to $f_{s}=\chi_{t} f_{\max }$, where $\chi_{t}=1 /\left(2 f_{\max } \Delta t\right)$ is the temporal oversampling factor, and approximately time-limited to $-p_{f} \Delta t<t<p_{f} \Delta t, 5 \leq p_{f} \leq 10$ (see [28] regarding more details about the APS function). This allows splitting $\mathbf{E}\left(\mathbf{r}_{n}, t\right)$ into $N_{l}^{v}$ consecutive bandlimited "sub-fields", $\mathbf{E}^{l}\left(\mathbf{r}_{n}, t\right)$, as

$$
\mathbf{E}\left(\mathbf{r}_{n}, t\right)=\sum_{l}^{N_{l}^{v}} \mathbf{E}^{l}\left(\mathbf{r}_{n}, t\right)=\sum_{l}^{N_{l}^{v}} \sum_{j=(l-1) M^{v}+1}^{l M^{v}} \mathbf{E}\left(\mathbf{r}_{n}, t_{j}\right) T_{j}^{A P S}(t) .
$$

Here, $N_{l}^{v} M^{v}=N_{t}, M^{v}$ is chosen such that the duration of each sub-field, $T^{v}=\left(M^{v}+2 p_{f}\right) \Delta t$, satisfies the condition $T^{v}<\left(R_{c, \alpha \alpha^{\prime}}-2 R^{v}\right) / c_{b}$, and $T_{j}^{A P S}(t)=T^{A P S}(t-j \Delta t)$ is the shifted APS function. Next, the potential (causal contribution) at element center $\mathbf{r}_{m}$ due to the $l$ th sub-fields of all source elements that reside in box $\alpha, \mathbf{P}_{c}^{l}\left(\mathbf{r}_{m}, t\right)$, is computed by

$$
\mathbf{P}_{c}^{l}\left(\mathbf{r}_{m}, t\right)=\sum_{p=0}^{K^{v}} \sum_{q=-K^{v}}^{K^{v}} \delta\left[t-\hat{\mathbf{k}}_{p q}^{v} \cdot\left(\mathbf{r}_{m}-\mathbf{r}_{\alpha^{\prime}}^{c}\right) / c_{b}\right] * \mathbf{G}_{l, \alpha^{\prime}}^{-}\left(\hat{\mathbf{k}}_{p q}^{v}, t\right)
$$

where

$$
\begin{gathered}
\mathbf{G}_{l, \alpha^{\prime}}^{-}\left(\hat{\mathbf{k}}_{p q}^{v}, t\right)=\mathcal{T}\left(\hat{\mathbf{k}}_{p q}^{v}, t\right) * \mathbf{G}_{l, \alpha}^{+}\left(\hat{\mathbf{k}}_{p q}^{v}, t\right) \\
\mathbf{G}_{l, \alpha}^{+}\left(\hat{\mathbf{k}}_{p q}^{v}, t\right)=\sum_{n \in \alpha} \delta\left[t+\hat{\mathbf{k}}_{p q}^{v} \cdot\left(\mathbf{r}_{n}-\mathbf{r}_{\alpha}^{c}\right) / c_{b}\right] * w_{n} \mathbf{E}^{l}\left(\mathbf{r}_{n}, t\right) .
\end{gathered}
$$

Here, * denotes temporal convolution, $\delta[\cdot]$ is the Dirac function, $\mathbf{G}_{l, \alpha}^{+}\left(\hat{\mathbf{k}}_{p q}^{v}, t\right)$ and $\mathbf{G}_{l, \alpha^{\prime}}^{-}\left(\hat{\mathbf{k}}_{p q}^{v}, t\right)$ represent the outgoing ray 
in box $\alpha$ and incoming ray in box $\alpha^{\prime}$, respectively. $\hat{\mathbf{k}}_{p q}^{v}$, $p=0, \ldots, K^{v}, q=-K^{v}, \ldots, K^{v}$, represent directions of the outgoing/incoming rays with a total of $N_{k}^{v}=\left(K^{v}+1\right)\left(2 K^{v}+1\right)$ directions, and $K^{v}=\left\lfloor 4 \pi \chi_{s} f_{s} R^{v} / c_{b}\right\rfloor+1$ is the number of spherical harmonics with the spherical oversampling factor $\chi_{s}$. In (15), the translation function $\mathcal{T}\left(\hat{\mathbf{k}}_{p q}^{v}, t\right)$ is

$$
\mathcal{T}\left(\hat{\mathbf{k}}_{p q}^{v}, t\right)=\frac{-w_{p q} \partial_{t}}{4 \pi R_{c, \alpha \alpha^{\prime}}} \sum_{k=0}^{K^{v}}(2 k+1) \Phi_{k}\left(\frac{c_{b} t}{R_{c, \alpha \alpha^{\prime}}}\right) \Phi_{k}\left(\frac{\hat{\mathbf{k}}_{p q}^{v} \cdot \mathbf{R}_{c, \alpha \alpha^{\prime}}}{R_{c, \alpha \alpha^{\prime}}}\right)
$$

where $\Phi_{k}(\cdot)$ is the Legendre polynomial of degree $k, w_{p q}$ are quadrature weights on the unit sphere [29], and $|t| \leq R_{c, \alpha \alpha^{\prime}} / c_{b}$.

The PWTD-accelerated computation of $\mathbf{P}_{c}^{l}\left(\mathbf{r}_{m}, t\right)$ in (14)-(16) is carried out in three stages. First, outgoing rays $\mathbf{G}_{l, \alpha}^{+}\left(\hat{\mathbf{k}}_{p q}^{v}, t\right)$ associated with box $\alpha$ are constructed by projecting the sub-field $\mathbf{E}^{l}\left(\mathbf{r}_{n}, t\right), n \in \alpha$ along directions $\hat{\mathbf{k}}_{p q}^{v}$ using (16). Note that the outgoing ray $\mathbf{G}_{l, \alpha}^{+}\left(\hat{\mathbf{k}}_{p q}^{v}, t\right)$ has three Cartesian components that can be independently constructed from the Cartesian components of $\mathbf{E}^{l}\left(\mathbf{r}_{n}, t\right)$. Next, the outgoing rays $\mathbf{G}_{l, \alpha}^{+}\left(\hat{\mathbf{k}}_{p q}^{v}, t\right)$ are translated into incoming rays associated with box $\alpha^{\prime}, \mathbf{G}_{l, \alpha^{\prime}}^{-}\left(\hat{\mathbf{k}}_{p q}^{v}, t\right)$, by convolving $\mathbf{G}_{l, \alpha}^{+}\left(\hat{\mathbf{k}}_{p q}^{v}, t\right)$ with the translation function $\mathcal{T}\left(\hat{\mathbf{k}}_{p q}^{v}, t\right)$ using (15). Finally, the causal potential contribution $\mathbf{P}_{c}^{l}\left(\mathbf{r}_{m}, t\right)$ are computed by projecting the incoming rays $\mathbf{G}_{l, \alpha^{\prime}}^{-}\left(\hat{\mathbf{k}}_{p q}^{v}, t\right)$ onto the observer element $m$ and summing over all directions using (14). Note that in (14)-(16), $\mathbf{P}_{c}^{l}\left(\mathbf{r}_{m}, t\right)$ is computed by decoupling its three Cartesian components and evaluating them independently using the scalar-field PWTD scheme. In practice, only outgoing/incoming rays of boxes at the finest level are constructed/projected directly from/onto the elements using (16) $/(14)$, those at higher levels are computed by a scalar spherical interpolation/filtering scheme described in [30].

The computational and memory costs of the PWTD-accelerated PC-based TD-EFVIE solver described in this and previous sections are briefly summarized next. Note that the analysis in [29] showed that the computational costs of spherical interpolation/filtering and translation operations for one ray in one box along all directions scale as $O\left(T^{v} N_{k}^{v} \log T^{v}\right)$ As there exist $N_{l}^{v}$ rays and $N_{g}^{v}$ boxes at each level, the overall computational costs of the spherical interpolation/filtering and translation scale as $\sum_{v=1}^{N_{L}} N_{k}^{v} N_{l}^{v} N_{g}^{v} T^{v} \log T^{v}=O\left(N_{s} N_{t}\right)$. Meanwhile, the computational costs of the construction/projection of outgoing/incoming rays at the finest level, near field calculation, and the evaluation of the electric field via finite differences, all scale as $O\left(N_{s} N_{t}\right)$. Therefore, the overall computational cost of the proposed PWTD-PC-EFVIE solver scales as $O\left(N_{s} N_{t}\right)$.

The memory cost of storing one outgoing/incoming ray in one box along all directions scales as $O\left(T^{v} N_{k}^{v}\right)$. As there are $O(1)$ rays in $N_{g}^{v}$ boxes at each level that need to be stored, the memory cost for storing the ray data scales as $\sum_{v=1}^{N_{L}} N_{g}^{v} T^{v} N_{k}^{v} O(1)=O\left(N_{s} \log N_{s}\right)$. On the other hand, storing the electric fields $\mathbf{E}_{n, j}$ for evaluation of the near field potential contributions in (7) and construction of level 1 outgoing rays in

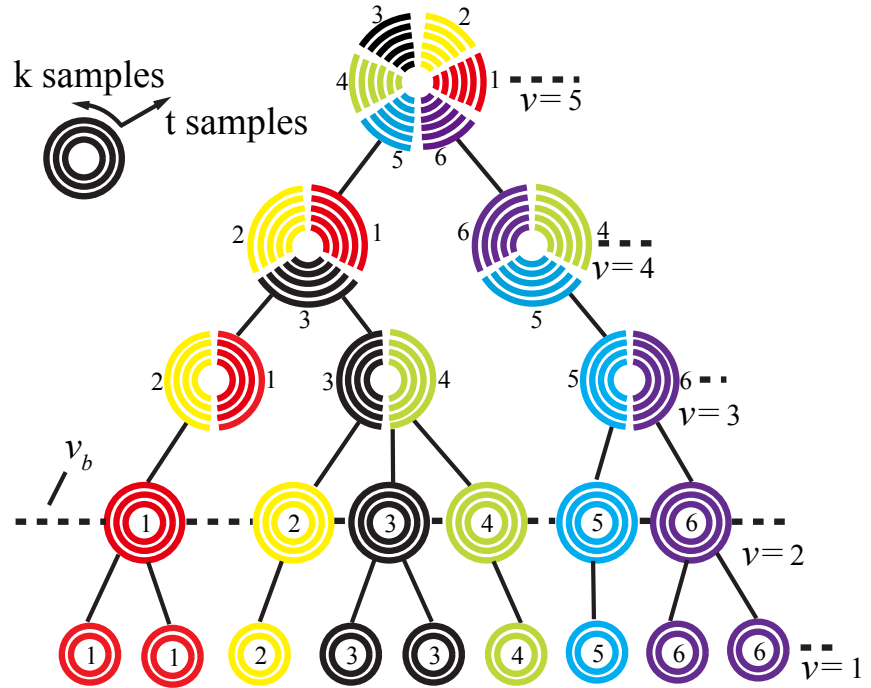

Fig. 2. Partitioning of boxes and their ray data in a five-level PWTD tree among six processors.

(16) requires only $O\left(N_{s}\right)$ memory. Hence, the overall memory cost of the PWTD-PC-EFVIE solver scales as $O\left(N_{s} \log N_{s}\right)$.

\section{Parallelization OF THE PWTD-PC-EFVIE SOLVER}

Despite its attractive computational and memory cost estimates, the PWTD-PC-EFVIE solver described in the previous section, if implemented for serial execution on single CPU, still has limited applicability to analysis of electrically large transient phenomena that oftentimes involve scatterers discretized using millions of spatial elements. Here, a highly scalable parallel implementation of the solver for execution on distributed-memory CPU clusters is described. Note that the proposed solver has two computation phases at the predictor/corrector step, v.i.z., the computation of vector potentials by the PWTD algorithm (first phase) and the computation of electric fields by finite differences (second phase). In what follows, the parallelization of these phases are expounded.

Efficient parallelization of the potential computation requires uniform partitioning of the memory and computation workloads associated with outgoing/incoming rays (i.e., ray data) in the PWTD algorithm. However, this task is nontrivial due to the PWTD algorithm's heterogeneous tree structure, as at each level the ray data is computed for $N_{g}^{v}=O\left(N_{s} / 8^{v}\right)$ boxes in spatial dimension, $N_{k}^{v}=O\left(4^{v}\right)$ samples in angular dimension, and $T^{v}=O\left(2^{v}\right)$ samples in temporal dimension; partitioning along a single dimension leads to poor load balance at either higher or lower PWTD tree levels [31]. Therefore, the proposed partitioning strategy distributes the loads hierarchically in more than one dimension depending on the number of groups $N_{g}^{v}$ at each level and the number of processors $N_{p}$. Let $v_{b}$ denote the highest possible level at which $N_{g}^{v} \geq N_{p}$. At level $v \leq v_{b}$, each processor stores the ray data for all angular and temporal samples in approximately $N_{g}^{v} / N_{p}$ boxes; at level $v>v_{b}$, each processor stores $N_{k}^{v} N_{g}^{v} / N_{p}$ angular samples and all temporal samples for one box. As an example, 
consider a five-level PWTD tree that is partitioned among six processors (Fig. 2). In Fig. 2, each set of concentric circles represents one box and its associated ray data. The angular and radial dimensions of the circles correspond to the angular and temporal samples of the ray data, respectively. The number shown near the concentric circles and arcs indicates the ID of the processor in charge of the data marked with a certain color. For this example, $N_{g}^{v}=9,6,3,2,1$ for $v=1, \ldots, 5$, and $N_{p}=6$, hence $v_{b}=2$. Each processor stores the complete ray data in one or two boxes at level $v=1,2$, and 1/2,1/3,1/6 portion of the angular samples of the ray data in one box at level $v=3,4,5$. This parallelization strategy results in computation and memory load balancing and produces scalable communication patterns among processors at all levels of the PWTD tree [31]. The computation loads of the PWTD stages are parti-

TABLE I

EXECUTION TIMES AND PARALLEL EFFICIENCIES OF THE PREDICTOR AND CORRECTOR STEPS

\begin{tabular}{|c|c|c|c|}
\hline \multicolumn{4}{|c|}{ Cube } \\
\hline & Predictor & Corrector & Overall \\
\hline$N_{p}=64$ & $17.87 \mathrm{~h}$ & $38.6 \mathrm{~min}$ & $18.76 \mathrm{~h}$ \\
\hline$N_{p}=1024$ & $1.35 \mathrm{~h}$ & $3.7 \mathrm{~min}$ & $1.44 \mathrm{~h}$ \\
\hline$\kappa$ & $82.7 \%$ & $65.7 \%$ & $81.7 \%$ \\
\hline \multicolumn{4}{|c|}{ Sphere } \\
\hline & Predictor & Corrector & Overall \\
\hline$N_{p}=128$ & $17.4 \mathrm{~h}$ & $1.3 \mathrm{~h}$ & $19 \mathrm{~h}$ \\
\hline$N_{p}=2048$ & $1.3 \mathrm{~h}$ & $8.3 \mathrm{~min}$ & $1.74 \mathrm{~h}$ \\
\hline$\kappa$ & $83.6 \%$ & $60 \%$ & $80.5 \%$ \\
\hline
\end{tabular}

tioned as follows:

- Construction/projection of outgoing/incoming rays at the finest level. Each processor constructs outgoing rays using (16) for the source elements in its $N_{g}^{1} / N_{p}$ boxes; similarly, it computes the partial causal potential contribution (due to PWTD) by projecting the incoming rays in (14) onto the observer elements in its $N_{g}^{1} / N_{p}$ boxes.

- Construction/projection of outgoing/incoming rays via spherical interpolation/filtering. At level $v \leq v_{b}$, each processor spherically interpolates/filters the complete ray data for its $N_{g}^{v} / N_{p}$ boxes; at level $v>v_{b}$, each processor spherically interpolates/filters the ray data for $O\left(T^{v} N_{g}^{v} / N_{p}\right)$ temporal samples and all angular samples of the ray data of one box. In other words, the main computation workloads are split along the spatial and temporal dimensions.

- Translation. At level $v \leq v_{b}$, each processor performs translation in all directions for the $N_{g}^{v} / N_{p}$ observations boxes it is responsible for; at level $v>v_{b}$, each processor carries out translation at $N_{k}^{v} N_{g}^{v} / N_{p}$ directions for the observer box it is in charge of. In other words, the computation workloads are distributed along the spatial and angular dimensions.

- Near field calculation. Each processor computes the noncausal potential contribution $\mathbf{P}_{n c}\left(\mathbf{r}_{m}, t_{i+1}\right)$ and partial caus-

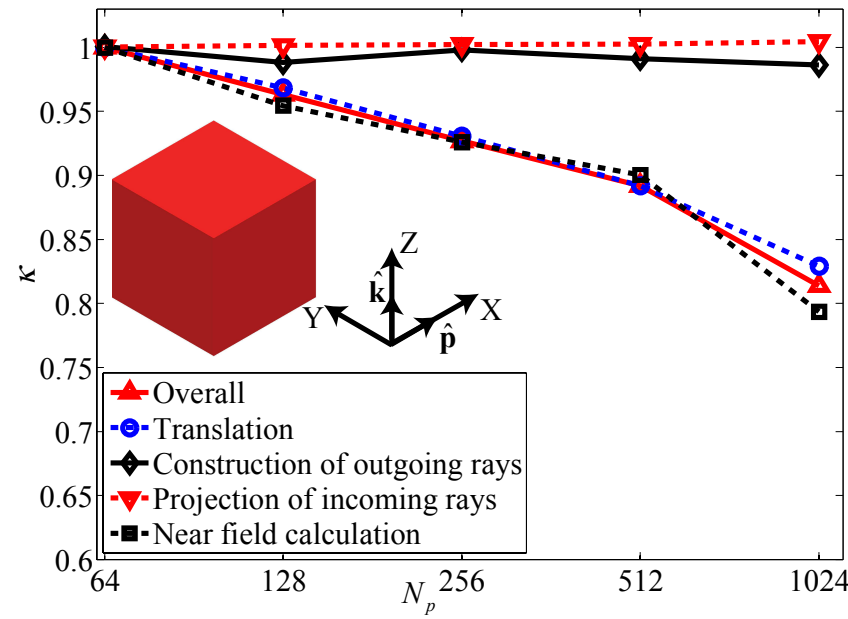

(a)

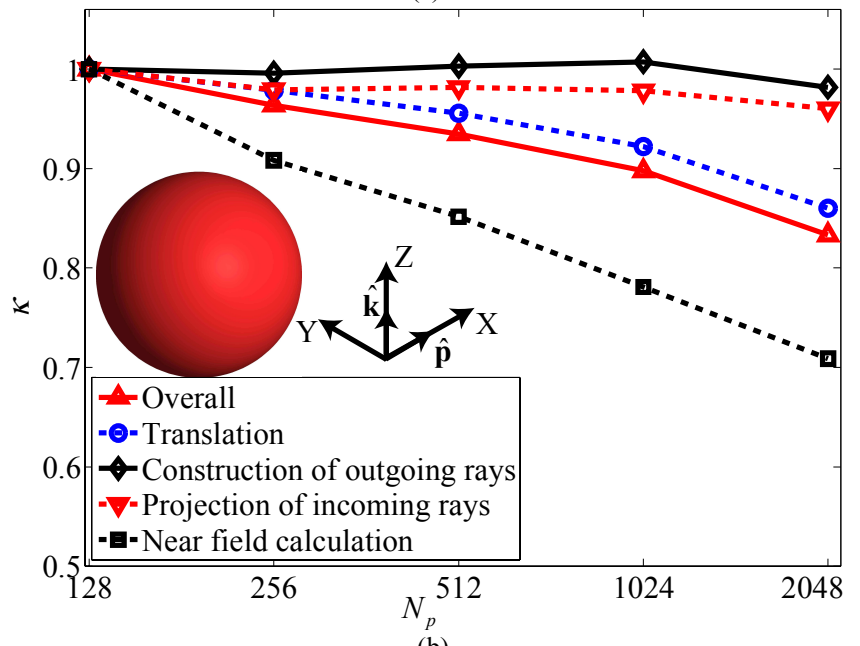

(b)

Fig. 3. Parallel efficiencies $\kappa$ of different PWTD stages applied at the predictor and corrector steps for the examples involving (a) a dielectric cube and (b) a dielectric sphere.

al potential contribution $\mathbf{P}_{c}\left(\mathbf{r}_{m}, t_{i+1}\right)$ for the observer elements in its $N_{g}^{1} / N_{p}$ boxes.

Next, the parallelization of the second phase is described. Note that the computation of electric field $\mathbf{E}\left(\mathbf{r}_{m}, t_{i}\right)$ at the $m$ th observer element in box $\alpha$ by the finite difference schemes in $(8) /(11)$ requires the vector potential $\mathbf{P}_{r}\left(\mathbf{r}, t_{i}\right)$ and $\mathbf{P}_{r}\left(\mathbf{r}, t_{i-1}\right)$ at its neighboring elements, which resides in box $\alpha$ and its adjacent boxes (Fig. 1). Therefore, the second computation phase can be easily parallelized using a partitioning strategy similar to that used in the near field calculation stage of the PWTD algorithm: each processor computes the electric fields at the observer elements in its $N_{g}^{v} / N_{p}$ boxes.

\section{NuMERICAL RESUltS}

This section presents several numerical examples that demonstrate the efficiency, accuracy, and applicability of the proposed parallel PWTD-PC-EFVIE solver. In all examples considered here, scatterers are excited by a plane wave with electric field 


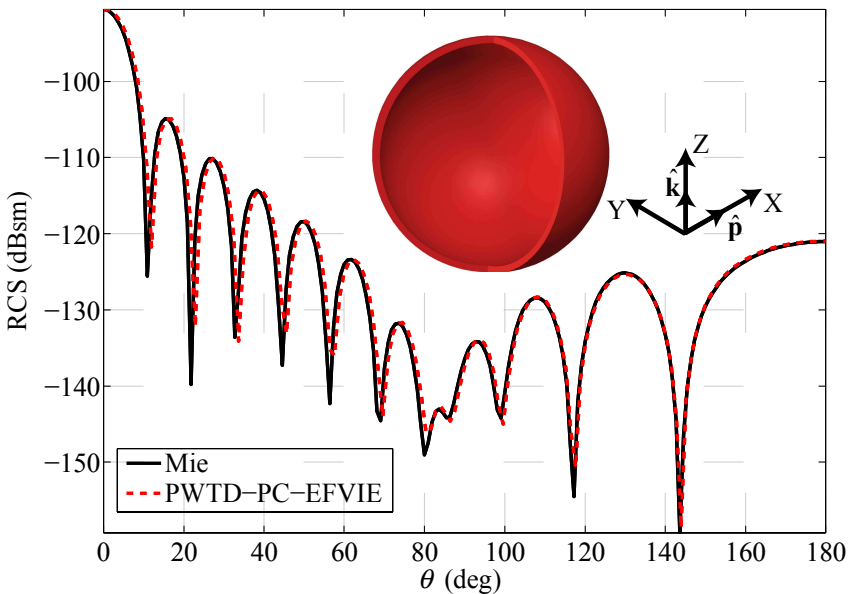

Fig. 4. Bistatic RCS of the dielectric shell computed at $299.4 \mathrm{THz}$ for $\phi=0^{\circ}$ and $\theta=[0,180]^{\circ}$ from the Mie series solution and the time domain data generated by the PWTD-PC-EFVIE solver.

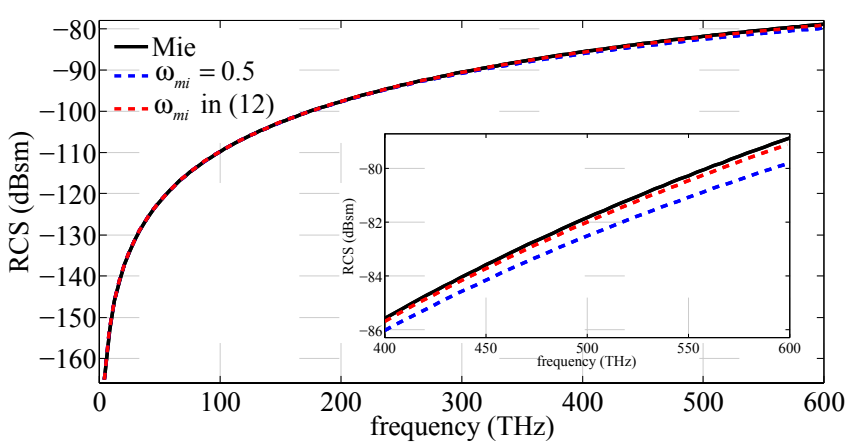

Fig. 5. Broadband RCS of the dielectric shell along $+z$ direction $(\theta=0)$ computed from the Mie series solution and the time domain data generated by the PWTD-PC-EFVIE solver.

$$
\begin{aligned}
& \mathbf{E}^{i}(\mathbf{r}, t)=\hat{\mathbf{p}} G\left(t-\mathbf{r} \cdot \hat{\mathbf{k}} / c_{b}\right) \\
& G(t)=\cos \left[2 \pi f_{0}\left(t-t_{0}\right)\right] e^{-\left(t-t_{0}\right)^{2} / 2 \sigma^{2}} .
\end{aligned}
$$

Here, $\hat{\mathbf{p}}$ and $\hat{\mathbf{k}}$ are polarization and propagation directions of the plane wave and $G(t)$ is a Gaussian pulse with central (modulation) frequency $f_{0}$, duration $\sigma$, and delay $t_{0}$. An essential bandwidth is selected as $f_{\mathrm{bw}}=3 /(2 \pi \sigma)$; this choice ensures that $99.998 \%$ of $G(t)$ ' energy resides within the frequency band $\left[f_{0}-f_{\mathrm{bw}} f_{0}+f_{\mathrm{bw}}\right]$. Consequently, the maximum frequency of $G(t)$ can essentially be defined as $f_{\max }=f_{0}+f_{\text {bw }}$. In all examples, $\hat{\mathbf{p}}=\hat{\mathbf{x}}, \hat{\mathbf{k}}=\hat{\mathbf{z}}$, and $t_{0}=8 \sigma$. Additionally, unless otherwise stated, it is assumed that all scatterers reside in free space $\left(\varepsilon_{b}=\varepsilon_{0}\right)$ and their geometrical centers are at the origin. All simulations were executed on a 16-rack IBM Blue Gene/P cluster located at the King Abdullah University of Science and Technology (KAUST) Supercomputing Laboratory (KSL). Each rack contains 1024 quad-core, 32-bit, $850 \mathrm{MHz}$ PowerPC compute nodes with 4GB memory and nodes are interconnected by a three-dimensional point-to-point "torus" network. The PWTD-PC-EFVIE solver leverages a hybrid MPI and OpenMP parallelization strategy; one MPI process is launched per CPU
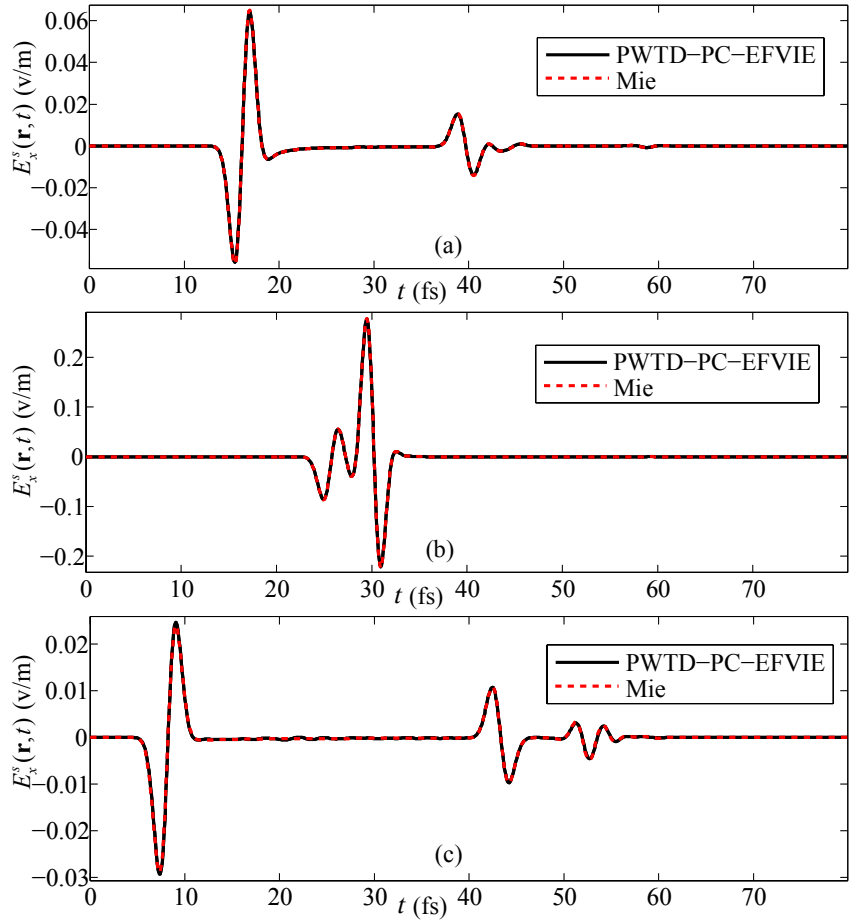

Fig. 6. $\hat{\mathbf{x}}$-components of the electric field scattered from the dielectric shell computed by the PWTD-PC-EFVIE solver and by inverse Fourier transforming Mie series solution at points (a) $(0,3 \mu \mathrm{m}, 0)$, (b) $(0,0,3 \mu \mathrm{m})$ and (c) $(0,0$, $-3 \mu \mathrm{m})$.

and OpenMP uses four cores of each CPU.

\section{A. Parallel Efficiency}

This subsection investigates the parallel efficiencies of the PWTD-PC-EFVIE solver's different stages. Here, the parallel efficiency $\kappa$ is measured as $\kappa=N_{\text {ref }} T_{N_{\text {ref }}} / N_{p} T_{N_{p}}$, where $T_{N_{r e f}}$ and $T_{N_{p}}$ are the execution times on $N_{r e f}^{p}$ and $N_{p}$ processors, respectively. Note that $N_{\text {ref }}$ is chosen as the minimum number of processors required to execute a given simulation in a 'reasonable' time. Parallel-efficiency investigations are carried out using two different scatterers as described next.

1) Cube

First, the PWTD-PC-EFVIE solver is applied to the analysis of transient scattering from a dielectric cube of edge length $4.7 \mu \mathrm{m}$ and permittivity $\mathcal{\varepsilon}(\mathbf{r})=1.5 \varepsilon_{0}$. The cube is excited by the electric field given in (18) with $f_{0}=400 \mathrm{THz}$ and $f_{\max }=600 \mathrm{THz}$. The cube is discretized into $N_{s}=804,357$ cubic elements of edge length $\Delta s=0.05 \mu \mathrm{m}$ resulting in $N_{s}=804,357$ source and $N_{s}^{\prime}=857,375$ observer points $\Delta s=0.05 \mu \mathrm{m}$. The simulation is carried out for $N_{t}=240$ time steps with step size $\Delta t=0.167 \mathrm{fs}$ and temporal basis function order $p=1$. The averaging factor $\omega_{m i}$ is computed by setting $\tau_{1}=1.3 t_{0}$ and $\tau_{2}=1.5 t_{0}$ in (12). A six-level PWTD tree is constructed upon setting $\gamma=6$ and the edge length of the boxes at the finest level to $0.294 \lambda$.

The parallel efficiencies $\kappa$ and execution times $T_{N}$ and $T_{N_{p}}$ of the predictor and corrector steps of the 


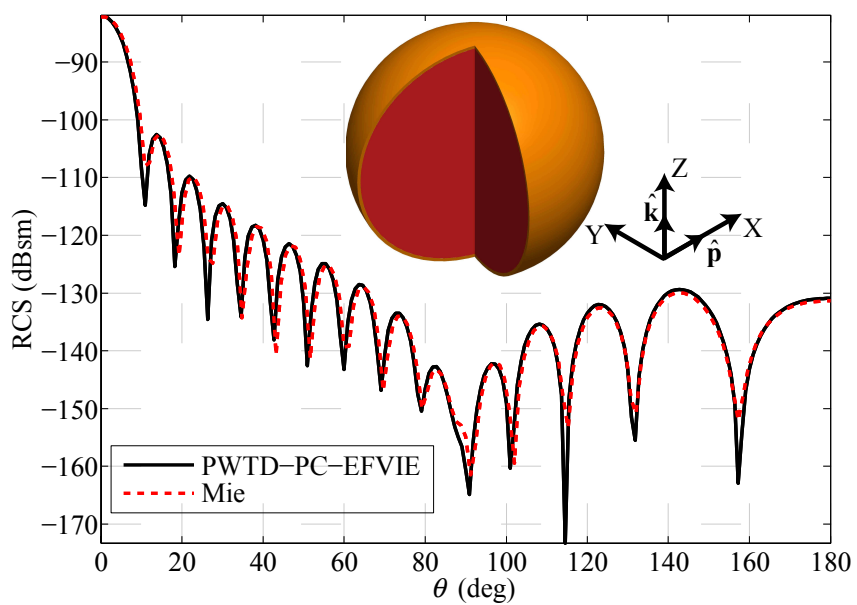

Fig. 7. Bistatic RCS of the two-layer sphere computed at $199.7 \mathrm{THz}$ for $\phi=0^{\circ}$ and $\theta=[0,180]^{\circ}$ from the Mie series solution and the time domain data generated by the PWTD-PC-EFVIE solver.

PWTD-PC-EFVIE solver, which are obtained for $N_{\text {ref }}=64$ and $N_{p}=1024$, are listed in Table I. It is clear that the parallel efficiency and execution time of the predictor step dominate those of the corrector step. This is simply because the calculation of the causal potential contribution $\mathbf{P}_{c}\left(\mathbf{r}_{m}, t_{i+1}\right)$, which is carried out at this step, is the computationally most dominant operation of the solver (as discussed in detail in Section II-B). Note that for this example, an overall efficiency of over $80 \%$ is achieved on $N_{p}=1024$ processors. Additionally, the parallel efficiencies $\kappa$ of the different PWTD stages applied to the computation of $\mathbf{P}_{n c}\left(\mathbf{r}_{m}, t_{i+1}\right)$ and $\mathbf{P}_{c}\left(\mathbf{r}_{m}, t_{i+1}\right)$ at the predictor and corrector steps are computed for $N_{\text {ref }}=64$ and $N_{p}$ varied from 64 to 1024. Fig. 3(a) plots overall $\kappa$ of the PWTD scheme and those of its four stages vs. $N_{p}$. Clearly, the parallel efficiency of the PWTD scheme is dominated by those of the translation and near field calculation stages.

\section{2) Sphere}

Next, the proposed solver is applied to the analysis of transient scattering from a dielectric sphere of radius $3 \mu \mathrm{m}$ and permittivity $\varepsilon(\mathbf{r})=1.2 \varepsilon_{0}$. The sphere is excited by the electric field given in (18) with $f_{0}=400 \mathrm{THz}$ and $f_{\max }=600 \mathrm{THz}$. The sphere is discretized into $N_{s}=904,089$ cubic elements of edge length $\Delta s=0.025 \mu \mathrm{m}$ resulting in $N_{s}=904,089$ source and $N_{s}^{\prime}=973,283$ observer points. $\Delta s=0.025 \mu \mathrm{m}$ The simulation is carried out for $N_{t}=100$ time steps with step size $\Delta t=0.084 \mathrm{fs}$ and temporal basis function order $p=1$. The averaging factor $\omega_{m i}$ is computed by setting $\tau_{1}=1.3 t_{0}$ and $\tau_{2}=1.5 t_{0}$ in (12). A seven-level PWTD tree is constructed upon setting $\gamma=6$ and the edge length of the boxes at the finest level to $0.2 \lambda$.

The parallel efficiencies $\kappa$ and execution times of $T_{N}$ and $T_{N}$ of the predictor and corrector steps of the PWTD-PC-EFVIE solver, which are obtained for $N_{\text {ref }}=128$ and $N_{p}=2048$, are listed in Table I. Again, an overall efficiency of over $80 \%$ is achieved for the solver. The parallel efficiencies $\kappa$ of the PWTD stages applied at the predictor and
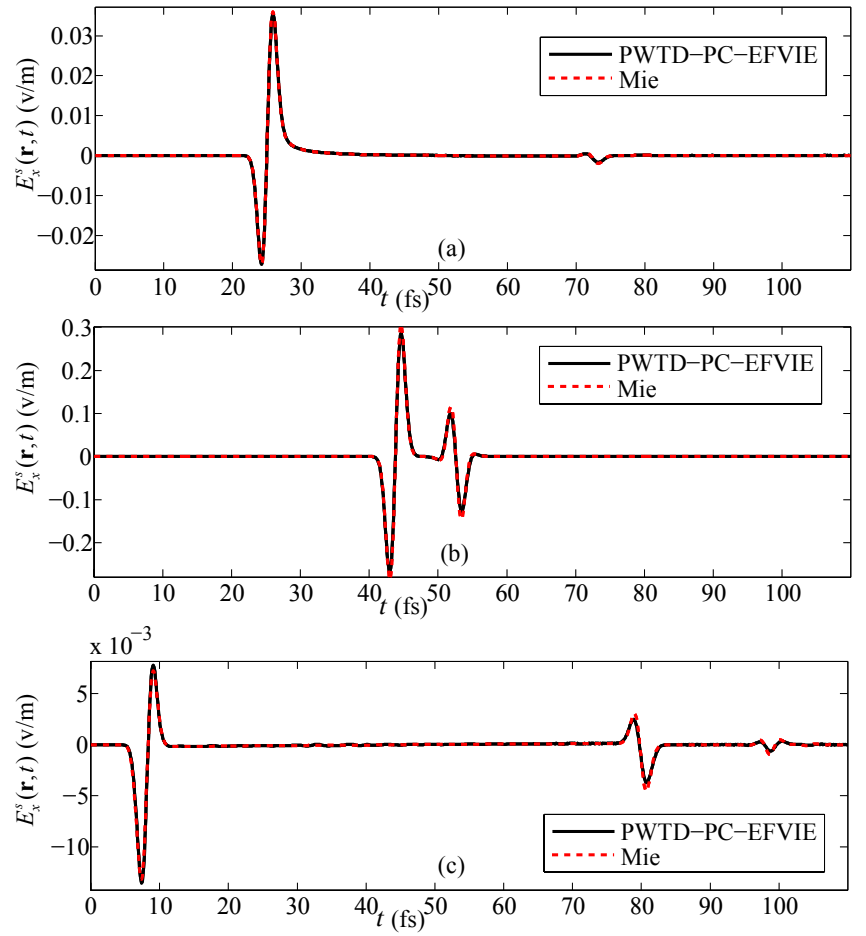

Fig. 8. $\hat{\mathbf{x}}$-components of the electric field scattered from the two-layer sphere computed by the PWTD-PC-EFVIE solver and by inverse Fourier transforming Mie series solution at points (a) $(5.7 \mu \mathrm{m}, 0,0)$, (b) $(0,0,5.7 \mu \mathrm{m})$ and (c) $(0$, $0,-5.7 \mu \mathrm{m})$.

corrector steps, which are obtained for $N_{\text {ref }}=128$ and $N_{p}$ varied from 128 to 2048, are plotted in Fig. 3(b).

\section{B. Canonical Examples}

This subsection demonstrates the accuracy of the PWTD-PC-EFVIE solver through its application to the computation of the far and near fields scattered from two canonical examples: a spherical shell and a two-layer sphere. In both examples, the radar cross section (RCS) of the scatterers (in frequency domain) is computed using the discrete Fourier transform of the time domain data generated by the solver. In addition, the $\hat{\mathbf{x}}$-component of the scattered field, $E_{x}^{s}(\mathbf{r}, t)$, is computed at several positions outside the scatterers. These results are compared with the Mie series solutions to quantify the accuracy of the solver in computing far and near fields.

1) Shell

First, the proposed solver is applied to the analysis of scattering from a dielectric shell with inner radius $2.55 \mu \mathrm{m}$, outer radius $2.7 \mu \mathrm{m}$, and permittivity $\mathcal{E}(\mathbf{r})=1.21 \varepsilon_{0}$. The shell is excited by the electric field given in (18) with $f_{0}=0 \mathrm{THz}$ and $f_{\max }=600 \mathrm{THz}$. The shell is discretized into $N_{s}=481,906$ cubic elements of edge length $\Delta s=0.03 \mu \mathrm{m}$ resulting in $N_{s}=481,906$ source and $N_{s}^{\prime}=770,828$ observer points $\Delta s=0.03 \mu \mathrm{m}$. It should be noted here that for shelled scatterers such as antenna radomes, the proposed solver requires significantly fewer number of spatial discretization elements as opposed to differential equation solvers that requires spatial discretization of the entire computation domain. The simulation 


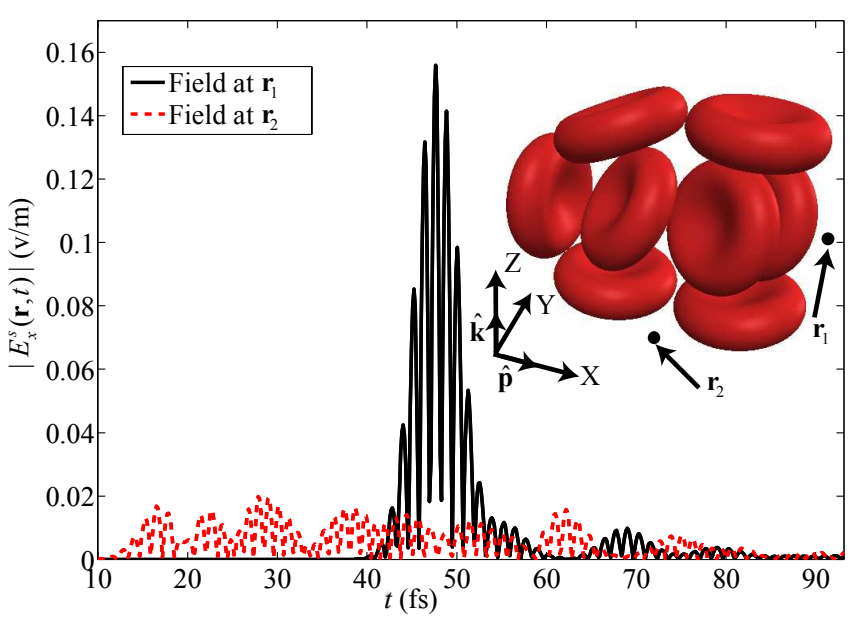

Fig. 9. Magnitudes of the $\hat{\mathbf{x}}$-component of the electric field scattered from the red blood cell aggregation computed by the PWTD-PC-EFVIE solver at points $\mathbf{r}_{1}=(19.3 \mu \mathrm{m}, 4 \mu \mathrm{m}, 7.5 \mu \mathrm{m})$ and $\mathbf{r}_{2}=(10 \mu \mathrm{m}, 4 \mu \mathrm{m}, 0)$.

is carried out for $N_{t}=800$ time steps with step size $\Delta t=0.1 \mathrm{fs}$ and temporal basis function order $p=1$. The averaging factor $\omega_{m i}$ is computed by setting $\tau_{1}=3.2 t_{0}$ and $\tau_{2}=3.5 t_{0}$ in (12). A six-level PWTD tree is constructed upon setting $\gamma=5.5$ and the edge length of the boxes at the finest level to $0.341 \lambda$. The simulation is completed in approximately 4.5 hours (wall time) on $N_{p}=1024$ processors using about 250 GB of memory.

The bistatic RCS of the shell at 299.4 THz computed from the time domain data generated by the proposed solver is compared with the Mie series solution in Fig. 4. Excellent agreement is observed. As expected, the maximum value of the RCS is achieved along the forward direction (i.e., $\theta=0^{\circ}$ ). The broadband RCS along the forward direction is computed using the constant averaging factor $\omega_{m i}=0.5$ and the time-dependent averaging factor in (12), respectively (Fig. 5). The accuracy corresponding to high frequency components of the output data can be dramatically improved by leveraging the time-dependent averaging factor. Moreover, the scattered fields $E_{x}^{s}(\mathbf{r}, t)$ are computed at three points [represented as $(0,3 \mu \mathrm{m}$, $0),(0,0,3 \mu \mathrm{m})$ and $(0,0,-3 \mu \mathrm{m})$ in Cartesian coordinates] by the proposed solver. These fields are compared to the inverse
Fourier transformed Mie series solutions in Fig. 6. The results agree well. Note that these fields consist of contributions resulting from refraction, reflection, and diffraction of electromagnetic waves.

2) Two-layer Sphere

Next, the proposed solver is applied to the analysis of scattering from a two-layer sphere with inner radius $5.25 \mu \mathrm{m}$ and outer radius $5.4 \mu \mathrm{m}$. The permittivities of the inner and outer layers are $\varepsilon(\mathbf{r})=1.02 \varepsilon_{0}$ and $\varepsilon(\mathbf{r})=1.08 \varepsilon_{0}$, respectively. The structure is excited by the electric field in (18) with $f_{0}=0 \mathrm{THz}$ and $f_{\max }=600 \mathrm{THz}$. The structure is discretized into $N_{s}=24,427,317$ cubic elements of edge length $\Delta s=0.03 \mu \mathrm{m}$ resulting in $N_{s}=24,427,317$ source and $N_{s}^{\prime}=25,042,247$ observer points. $\Delta s=0.03 \mu \mathrm{m}$ The simulation is carried out for $N_{t}=1000$ time steps with step size $\Delta t=0.1 \mathrm{fs}$. The averaging factor $\omega_{m i}$ is computed by setting $\tau_{1}=3 t_{0}$ and $\tau_{2}=3.5 t_{0}$ in (12). A seven-level PWTD tree is constructed upon setting $\gamma=5$ and the edge length of the boxes at the finest level to $0.34 \lambda$. The simulation is completed in 15.5 hours (wall time) on $N_{p}=4096$ using about 1.6 TB of memory. To the best of authors' knowledge, this is the largest problem ever solved using TD-VIE solvers. Although other state-of-art frequency-domain VIE solvers can handle even larger scattering problems $[32,33]$, it's worth noting that the proposed TD-VIE solver can obtain broadband data in one simulation as opposed to frequency-domain VIE solvers that require one simulation per frequency sample.

The bistatic RCS of the two-layer sphere at $199.7 \mathrm{THz}$ computed from the time domain data generated by the proposed solver is compared with the Mie series solution in Fig. 7. The results show good agreement. Fig. 8 plots the scattered fields $E_{x}^{s}(\mathbf{r}, t)$ computed at $(5.7 \mu \mathrm{m}, 0,0),(0,0,5.7 \mu \mathrm{m})$ and $(0,0$, $-5.7 \mu \mathrm{m})$ by the proposed solver and by inverse Fourier transforming the Mie series solution. Again, good agreement is achieved demonstrating the accuracy of the proposed solver.

\section{Real-Life Example: Red Blood Cell Aggregation}

Finally, the applicability of the PWTD-PC-EFVIE solver is demonstrated through its application to the transient analysis of electromagnetic wave interactions with a red blood cell aggregation. The aggregation consists of eight red blood cells and

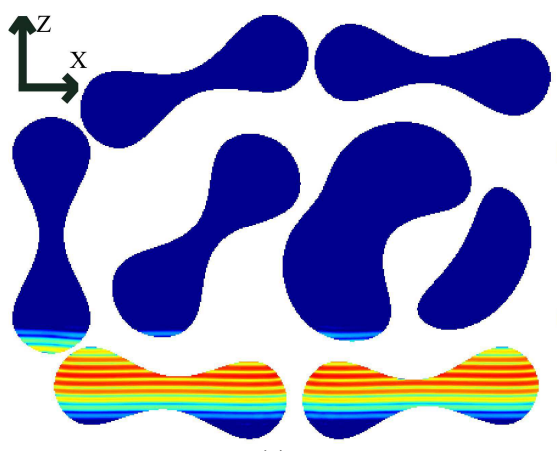

(a)

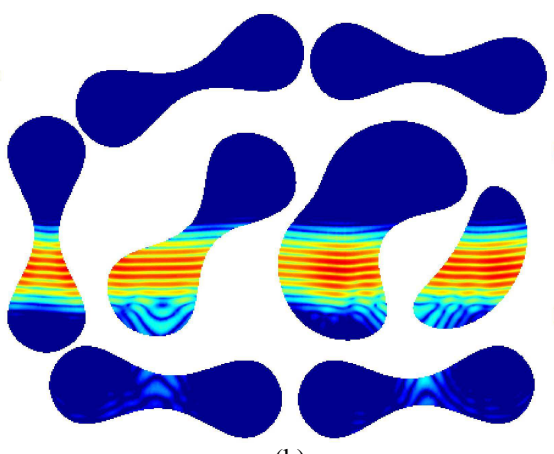

(b)

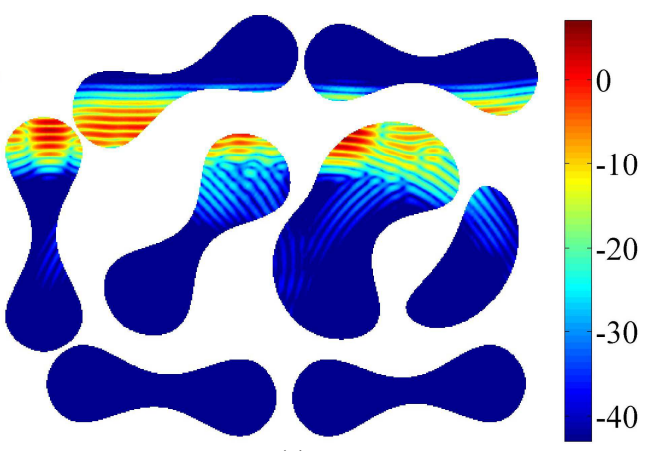

(c)

Fig. 10. Snapshots of the total electric fields (in dB) induced in the red blood cell aggregation computed by PWTD-PC-EFVIE solver at (a) $t=220 \Delta t,(b)$ $t=340 \Delta t$, (c) $t=500 \Delta t$. 
fits in a fictitious box with dimensions $18.3 \mu \mathrm{m} \times 8.7 \mu \mathrm{m} \times 14.5 \mu \mathrm{m}$ (Fig. 9). The geometrical details of each cell are described in [11]. The permittivities of the cells and the background medium are $\varepsilon(\mathbf{r})=1.97 \varepsilon_{0}$ and $\varepsilon_{b}=1.81 \varepsilon_{0}$, respectively. The aggregation is excited by the electric field given in (18) with $f_{0}=400 \mathrm{THz}$ and $f_{\max }=600 \mathrm{THz}$. The cells are discretized into $N_{s}=11,746,563$ cubic elements of edge length $\Delta s=0.03 \mu \mathrm{m}$ resulting in $N_{s}=11,746,563$ source and $N_{s}^{\prime}=13,775,837$ observer points. $\Delta s=0.03 \mu \mathrm{m}$ The simulation is carried out for $N_{t}=700$ time steps with step size $\Delta t=0.134 \mathrm{fs}$ and temporal basis function order $p=1$. The averaging factor $\omega_{m i}$ is computed by setting $\tau_{1}=1.3 t_{0}$ and $\tau_{2}=1.6 t_{0}$ in (12). An eight-level PWTD tree is constructed upon setting $\gamma=6$ and the edge length of the boxes at the finest level to $0.375 \lambda$ The simulation is completed in 23.5 hours (wall time) on $N_{p}=4096$ processors using about 1.2 TB of memory.

Fig. 9 plots the magnitude of the scattered field's $\hat{\mathbf{x}}$ component, $\left|E_{x}^{s}(\mathbf{r}, t)\right|$, computed by the proposed solver at points $(19.3 \mu \mathrm{m}, 4 \mu \mathrm{m}, 7.5 \mu \mathrm{m})$ and $(10 \mu \mathrm{m}, 4 \mu \mathrm{m}, 0)$. Furthermore, Fig. 10 provides the snapshots of the magnitude of the total electric field $\mathbf{E}(\mathbf{r}, t)$ induced inside the cells upon excitation (i.e., illumination under light). The snapshots are taken on the $y=4 \mu \mathrm{m}$ plane at times $220 \Delta t, 340 \Delta t$, and $500 \Delta t$. These results help better understand the diffraction and refraction phenomena pertinent to light incident on red blood cells.

\section{CONCLUSION}

This paper presents a PWTD-accelerated explicit MOT scheme for solving the TD-EFVIE to analyze transient scattering problems that involve electrically large inhomogeneous dielectric objects. The proposed scheme leverages simple pulse and Lagrange polynomials as spatial and temporal basis functions to discretize electric flux density, and employs a finite difference scheme to compute electric fields from magnetic vector potentials. An improved predictor-corrector scheme is proposed to maintain the stability of the solver. The computation of the vector potentials are accelerated by a scalar-field PWTD scheme, which reduces the overall memory and computational cost of the solver from $O\left(N_{s}^{4 / 3}\right)$ and $O\left(N_{t} N_{s}^{2}\right)$ to $O\left(N_{s} \log N_{s}\right)$ and $O\left(N_{t} N_{s}\right)$, respectively. In addition, this PWTD-PC-EFVIE solver is parallelized on distributed-memory clusters by employing a hierarchical partitioning strategy that yields favorable load balance and parallel efficiencies when the number of processors is large. The parallelized solver is applied to the analysis of transient scattering from canonical objects that are discretized using 25 million spatial basis functions, which to the authors' knowledge, is the largest scattering problem ever simulated using TD-VIE solvers. In addition, the proposed solver is applied to the transient electromagnetic characterizations of a red blood cell aggregation that is discretized using 12 million spatial basis functions.

\section{ACKNOWLEDGMENT}

The authors would like to thank the King Abdullah University of Science and Technology (KAUST) Supercomputing Laboratory (KSL) for providing the required computational resources.

\section{REFERENCES}

[1] R. U. Nair and R. M. Jha, "Electromagnetic design and performance analysis of airborne radomes: trends and perspectives," IEEE Antennas Propag. Mag., vol. 56, pp. 276-298, 2014.

[2] O. Ergul, A. Arslan-Ergul, and L. Gurel, "Computational study of scattering from healthy and diseased red blood cells," J. Biomed. Opt., vol. 15, pp. 045004-045004-8, 2010.

[3] N. T. Gres, A. A. Ergin, E. Michielssen, and B. Shanker, "Volume-integral-equation-based analysis of transient electromagnetic scattering from three-dimensional inhomogeneous dielectric objects," Radio Sci., vol. 36, pp. 379-386, 2001.

[4] B. Shanker, K. Aygun, and E. Michielssen, "Fast analysis of transient scattering from lossy inhomogeneous dielectric bodies," Radio Sci., vol. 39, 2004.

[5] G. Kobidze, J. Gao, B. Shanker, and E. Michielssen, "A fast time domain integral equation based scheme for analyzing scattering from dispersive objects," IEEE Trans. Antennas Propag., vol. 53, pp. 1215-1226, 2005.

[6] Y. Shi and J.-M. Jin, "A time-domain volume integral equation and its marching-on-in-degree solution for analysis of dispersive dielectric objects," IEEE Trans. Antennas Propag., vol. 59, pp. 969-978, 2011.

[7] S. Bin Sayed, H. A. Ulku, and H. Bagci, "A stable marching on-in-time scheme for solving the time-domain electric field volume integral equation on high-contrast scatterers," IEEE Trans. Antennas Propag., vol. 63, pp. 3098-3110, 2015.

[8] D. H. Schaubert, D. R. Wilton, and A. W. Glisson, "A tetrahedral modeling method for electromagnetic scattering by arbitrarily shaped inhomogeneous dielectric bodies," IEEE Trans. Antennas Propag., vol. 32, pp. 77-85, 1984.

[9] G. Manara, A. Monorchio, and R. Reggiannini, "A space-time discretization criterion for a stable time-marching solution of the electric field integral equation," IEEE Trans. Antennas Propag., vol. 45, pp. 527-532, 1997.

[10] D. S. Weile, G. Pisharody, N.-W. Chen, B. Shanker, and E. Michielssen, "A novel scheme for the solution of the time-domain integral equations of electromagnetics," IEEE Trans. Antennas Propag., vol. 52, pp. 283-295, 2004.

[11] Y. Beghein, K. Cools, H. Bagci, and D. De Zutter, "A space-time mixed Galerkin marching-on-in-time scheme for the time-domain combined field integral equation," IEEE Trans. Antennas Propag., vol. 61, pp. 1228-1238, 2013.

[12] A. J. Pray, Y. Beghein, N. V. Nair, K. Cools, H. Bagci, and B. Shanker, "A higher order space-time Galerkin scheme for time domain integral equations," IEEE Trans. Antennas Propag., vol. 62, pp. 6183-6191, 2014.

[13] G. Pisharody and D. S. Weile, "Robust solution of time-domain integral equations using loop-tree decomposition and bandlimited extrapolation," IEEE Trans. Antennas Propag., vol. 53, pp. 2089-2098, 2005.

[14] Y. Beghein, K. Cools, and F. P. Andriulli, "A DC stable and large-time step well-balanced TD-EFIE based on quasi-Helmholtz projectors," IEEE Trans. Antennas Propag., vol. 63, pp. 3087-3097, 2015.

[15] B. Shanker, M. Lu, J. Yuan, and E. Michielssen, "Time domain integral equation analysis of scattering from composite bodies via exact evaluation of radiation fields," IEEE Trans. Antennas Propag., vol. 57, pp. 1506-1520, 2009

[16] Y. Shi, M.-Y. Xia, R.-S. Chen, E. Michielssen, and M. Lu, "Stable electric field TDIE solvers via quasi-exact evaluation of MOT matrix elements," IEEE Trans. Antennas Propag., vol. 59, pp. 574-585, 2011.

[17] A. J. Pray, N. V. Nair, and B. Shanker, "Stability properties of the time domain electric field integral equation using a separable approximation for the convolution with the retarded potential," IEEE Trans. Antennas Propag., vol. 60, pp. 3772-3781, 2012.

[18] H. A. Ulku and A. A. Ergin, "Application of analytical retarded-time potential expressions to the solution of time domain integral equations," IEEE Trans. Antennas Propag., vol. 59, pp. 4123-4131, 2011.

[19] H. A. Ulku and F. Dikmen, "On the evaluation of retarded-time potentials for SWG bases," IEEE Antennas Wireless Propagat. Lett., vol. 10, pp. 187-190, 2011 
[20] M.-D. Zhu, X.-L. Zhou, and W.-Y. Yin, "Efficient evaluation of double surface integrals in time-domain integral equation formulations," IEEE Trans. Antennas Propag., vol. 61, p. 4653, 2013.

[21] S. J. Dodson, S. P. Walker, and M. J. Bluck, "Implicitness and stability of time domain integral equation scattering analyses," Appl. Comp Electromag. Soc. J., vol. 13, pp. 291-301, 1998.

[22] A. Al-Jarro, M. A. Salem, H. Bagci, T. M. Benson, P. Sewell, and A. Vukovic, "Explicit solution of the time domain volume integral equation using a stable predictor-corrector scheme," IEEE Trans. Antennas Propag., vol. 60, pp. 5203-5214, 2012.

[23] A. Al-Jarro, M. Cheeseman, and H. Bagci, "Distributed-memory parallelization of an explicit time-domain volume integral equation solver on Blue Gene/P," Appl. Comp. Electromag. Soc. J., vol. 27, pp. 132-144, 2012.

[24] S. Feki, A. Al-Jarro, A. Clo, and H. Bagci, "Porting an explicit time-domain volume-integral-equation solver on GPUs with OpenACC," IEEE Antennas Propag. Mag., vol. 56, pp. 265-277, 2014.

[25] B. Shanker, A. A. Ergin, M. Lu, and E. Michielssen, "Fast analysis of transient electromagnetic scattering phenomena using the multilevel plane wave time domain algorithm," IEEE Trans. Antennas Propag., vol. 51, pp. 628-641, 2003

[26] A. E. Yilmaz, J.-M. Jin, and E. Michielssen, "Time domain adaptive integral method for surface integral equations," IEEE Trans. Antennas Propag., vol. 52, pp. 2692-2708, 2004.

[27] J. Waldvogel, "The Newtonian Potential of Homogeneous Polyhedra," $J$. Appl. Math. Phys., vol. 30, pp. 388-398, 1979.

[28] J. Knab, "Interpolation of band-limited functions using the approximate prolate series," IEEE Trans. Inform. Theory, vol. 25, pp. 717-720, 1979.

[29] A. A. Ergin, B. Shanker, and E. Michielssen, "The plane-wave time-domain algorithm for the fast analysis of transient wave phenomena," IEEE Antennas Propag. Mag., vol. 41, pp. 39-52, 1999.

[30] R. Jakob-Chien and B. K. Alpert, "A fast spherical filter with uniform resolution," J. Comput. Phys., vol. 136, pp. 580-584, 1997.

[31] Y. Liu, H. Bagci, and E. Michielssen, "Progress in parallel implementation of the multilevel plane wave time domain algorithm," in Proc. IEEE Int. Symp. AP-S/URSI, 2013, pp. 48-48.

[32] F. Wei and A. E. Yilmaz, "A more scalable and efficient parallelization of the adaptive integral method-Part II: BIOEM application," IEEE Trans. Antennas Propag., vol. 62, pp. 727-738, 2014.

[33] M. A. Yurkin and A. G. Hoekstra, "The discrete-dipole-approximation code ADDA: capabilities and known limitations," J. Quant. Spectrosc. Radiat. Transfer vol. 112, pp. 2234-2247, 2011.

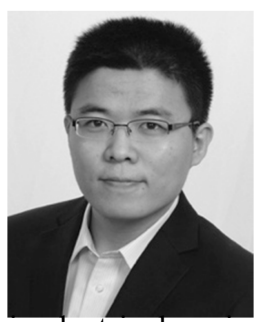

Yang Liu (S'11-M'15) received the B.S. degree in electrical engineering from Shanghai Jiao Tong University, Shanghai, China, in 2010. He received the M.S. degrees in electrical engineering and mathematics from the University of Michigan, Ann Arbor, MI, USA, in 2013 and 2014, respectively. He received the Ph.D. degree in electrical engineering from the University of Michigan, Ann Arbor, MI, USA, in 2015.

Since August 2010, he has been a graduate Research Assistant at the Radiation Laboratory, University of Michigan. Since June 2015, he has been working as a Research Fellow at the Radiation Laboratory, University of Michigan. His main research interest is in computational electromagnetics, with focus on fast time domain and frequency domain integral equation methods.

Dr. Liu authored the second place paper of the student paper competition of the 28th International Review of Progress in Applied Computational Electromagnetics, 2012. He also coauthored the first place paper of the student paper competition of the 12th International Workshop on Finite Elements for Microwave Engineering, 2014

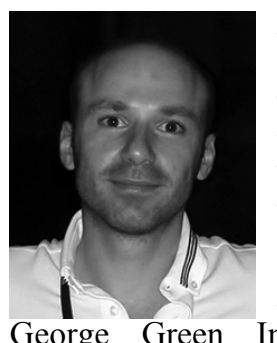

Ahmed Al-Jarro (M'11) received the B.Eng. degree in electronic engineering with Spanish and the Ph.D. degree in electrical and electronic engineering from the University of Nottingham, Nottingham, UK, in 2001 and 2004, respectively.

From 2004 to 2010, he was a Research Assistant and Research Fellow at the George Green Institute for Electromagnetics Research (GGIEMR), University of Nottingham, Nottingham, UK. In 2010, he worked as a Postdoctoral Research Fellow at the Division of Physical Sciences and Engineering, King Abdullah University of Science and Technology (KAUST), Thuwal, Saudi Arabia. In 2012, he was a Senior Research Associate at the Photonics Group, University College London, London, UK. In 2014, he joined Fujitsu Laboratories of Europe Ltd., London, UK, as a Principal Researcher at the Technical Computing Research Division. His research interests include computational electromagnetics for analyzing photonic and optical devices and the development of massively distributed and accelerated state-of-the-art computational algorithms.

Dr. Al-Jarro was the recipient of the EPSRC Knowledge Transfer Partnership in collaboration with Photon Design Ltd., Oxford, UK, from the University of Nottingham Research Innovation Services and University College London Advances Unit in 2010 and 2014, respectively. In 2010, he was the recipient of the Japan Society for the Promotion of Science (JSPS) Fellowship Award. In 2014, he received the UK National Supercomputing Services EPSRC Grant on ARCHER.

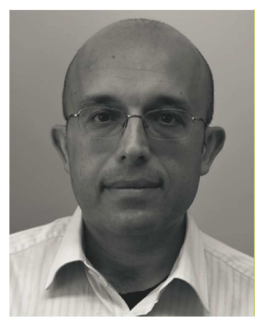

Hakan Bağcı (S'98-M'07-SM'14) received the B.S. degree in electrical and electronics engineering from the Bilkent University, Ankara, Turkey, in June 2001 and the M.S. and Ph.D. degrees in electrical and computer engineering from the University of Illinois at Urbana-Champaign (UIUC), Urbana, IL, USA, in August 2003 and January 2007, respectively.

From June 1999 to July 2001, he worked as an Undergraduate Researcher at the Computational Electromagnetics Group, Bilkent University. From August 2001 to December 2006, he was a Research Assistant at the Center for Computational Electromagnetics and Electromagnetics Laboratory,UIUC. From January 2007 to August 2009, he worked as a Research Fellow at the Radiation Laboratory, University of Michigan. In August 2009, he joined the Division of Physical Sciences and Engineering at the King Abdullah University of Science and Technology (KAUST), Thuwal, Saudi Arabia, as an Assistant Professor of electrical engineering. His research interests include various aspects of computational electromagnetics with emphasis on time domain integral equations and their fast marching-on-in-time-based solutions, well-conditioned integral-equation formulations, and development of fast hybrid methods for analyzing statistical EMC/EMI phenomena on complex and fully loaded platforms.

Dr. Bağc1 was the recipient of the 2008 International Union of Radio Scientists (URSI) Young Scientist Award and the 2004-2005 Interdisciplinary Graduate Fellowship from the Computational Science and Engineering Department, UIUC. 
His paper titled "Fast and Rigorous Analysis of EMC/EMI Phenomena on Electrically Large and Complex Structures Loaded With Coaxial Cables" was one of the three finalists (with honorable mention) for the 2008 Richard B. Schulz Best Transactions Paper Award given by the IEEE Electromagnetic Compatibility Society. He authored and coauthored three finalist papers and another paper, which is awarded honorable mention, in the student paper competitions at the 2005, 2008, and 2010 IEEE Antennas and Propagation Society International Symposiums.

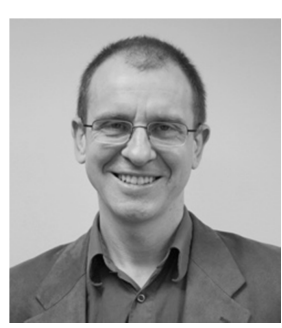

Eric Michielssen (M'95-SM'99-F'02) received the M.S. degree in electrical engineering (Summa Cum Laude) from the Katholieke Universiteit Leuven (KUL), Leuven, Belgium, in 1987, and the $\mathrm{Ph} . \mathrm{D}$. degree in electrical engineering from the University of Illinois at Urbana-Champaign (UIUC), Urbana, IL, USA, in 1992.

He joined the faculty of the UIUC Department of Electrical and Computer Engineering in 1993, reaching the rank of Full Professor in 2002. In 2005, he joined the University of Michigan (UM) as a Professor of Electrical Engineering and Computer Science. Since 2009, he has been directing the University of Michigan Computational Science Certificate Program. He authored or coauthored more than 160 journal papers and book chapters and more than 300 papers in conference proceedings. His research interests include all aspects of theoretical and applied computational electromagnetics. His research focuses on the development of fast frequency and time domain integral-equation-based techniques for analyzing electromagnetic phenomena, and the development of robust optimizers for the synthesis of electromagnetic/optical devices.

Dr. Michielssen received a Belgian American Educational Foundation Fellowship in 1988 and a Schlumberger Fellowship in 1990. Furthermore, he received a 1994 International Union of Radio Scientists (URSI) Young Scientist Fellowship, a 1995 National Science Foundation CAREER Award, and the 1998 Applied Computational Electromagnetics Society (ACES) Valued Service Award. In addition, he was named 1999 URSI United States National Committee Henry G. Booker Fellow and selected as the recipient of the 1999 URSI Koga Gold Medal. He also was awarded the UIUC's 2001 Xerox Award for Faculty Research, appointed 2002 Beckman Fellow in the UIUC Center for Advanced Studies, named 2003 Scholar in the Tel Aviv University Sackler Center for Advanced Studies, selected as UIUC 2003 University and Sony Scholar; in 2011 he received the UM College of Engineering David E. Liddle Research Excellence Award. He is a member of URSI Commission B. He served as the Technical Chairman of the 1997 Applied Computational Electromagnetics Society (ACES) Symposium (Review of Progress in Applied Computational Electromagnetics, March 1997, Monterey, CA, USA), and served on the ACES Board of Directors (1998-2001 and 20022003) and as ACES Vice-President (1998-2001). From 1997 to 1999, he was an Associate Editor for Radio Science, and from 1998 to 2008 he served as an Associate Editor for the IEEE TRANSACTIONS ON ANTENNAS AND PROPAGATION. 